\title{
Class A GPCRs: a multifaceted reality
}

\author{
Chiara Parravicini • Cristina Sensi • Ivano Eberini
}

Published online: 29 July 2011

(C) Springer Science+Business Media B.V. 2011

\section{Article summary}

The research group led by Ray Stevens has recently crystallized human $\mathrm{A}_{2 \mathrm{~A}}$ adenosine receptor $\left(\mathrm{A}_{2 \mathrm{~A}} \mathrm{AR}\right)$ bound to a pharmacological agonist, the compound UK-432097. This new high-resolution structure (RCSB ID: 3QAK) joins the already existing $\mathrm{A}_{2 \mathrm{~A}} \mathrm{AR}$ bound to the antagonist ZM2451385 (RCSB ID: 3EML), and enriches the variety of X-ray resolved, class A, G protein-coupled receptors (GPCRs): bovine and squid (rhod)opsins (Rh), turkey $\beta_{1}$ and human $\beta_{2}$-adrenergic receptors $\left(\beta_{1} \mathrm{AR}\right.$ and $\left.\beta_{2} \mathrm{AR}\right)$, human CXCR4 chemokine receptor and human D3 dopamine receptor (D3R). To shed light on the molecular mechanism of $\mathrm{A}_{2 \mathrm{~A}} \mathrm{AR}$ activation, the authors analyse in detail the agonist- and antagonist-bound structures, comparing binding modes and highlighting local changes, in an attempt to recognize the conformational modifications of the receptor at the very beginning of the signal transduction pathway. Authors describe at atomic level the ligandreceptor interaction network, identifying and evaluating all the residues involved in the molecular recognition process. They connect affinity and selectivity of the UK-432097 agonist to the very high number of interactions between ligand and receptor. Among these, they single out the ribose ring as determinant for discriminating between agonistic or

Highlight presented by Maria P. Abbracchio

Xu F, Wu H, Katritch V, Han GW, Jacobson KA, Gao ZG, Cherezov V, Stevens RC (2011) Structure of an agonist-bound human A2A adenosine receptor. Science 332:322-327; doi:10.1126/science.1202793

C. Parravicini $\cdot$ C. Sensi $\cdot$ I. Eberini $(\bowtie)$

Department of Pharmacological Sciences, University of Milan, Milan, Italy

e-mail: ivano.eberini@gmail.com antagonistic effects. They report how the binding of the UK-432097 activator induces a coordinated motion of helix VI, which rotates by approximately $30^{\circ}$ clockwise with respect to helix V; at the same time, helix VII exhibits a seesaw-like movement around the ribose ring. The authors point out that, while helices I to IV compose a rigid core, helices V, VI and VII are involved in macroscopic rearrangements with spring-like motions. The authors conclude the paper suggesting that, while other activators on different GPCRs shift the dynamic equilibrium between multiple receptor conformations, the agonist UK-432097 stabilizes only one receptor conformation.

\section{Commentary}

In our opinion, similar to the other few papers devoted to the characterization of class A GPCRs, the paper discussed in this commentary has a wider significance than the mere clarification of structure, molecular recognition mechanism, and operability of a single, specific receptor. Together, these reports represent instead the first steps toward the understanding of a common process, which should be the foundation of the most up-to-date research in pharmaceutical sciences. The large majority of currently marketed drugs indeed act through GPCRs, and have profoundly changed the medical approach to disease as well as patients' life quality and expectance.

Summing up data from papers devoted to class A GPCR structure since 2000 has allowed the scientific community to recognize some common features that are crucial for deciphering the operability of these proteins. However, the studies in this field have also revealed an unexpected heterogeneity and complexity in class A GPCR operability, which made it difficult to export the knowledgebase 
collected on a specific receptor to another. Several questions are pending, and the scientific community will have to make a great effort in order to solve them.

While discussing their structural data on $\mathrm{A}_{2 \mathrm{~A}} \mathrm{AR}$, Stevens and colleagues report the difficulty in crystallizing the receptor bound to an agonist in its active state without additional stabilization by a $\mathrm{G}$ protein or a mimetic. This is a common issue affecting all the crystallized class A GPCRs, except opsin, for which a ligand-free activated form exists independently from the binding to its cognate $G$ protein. An efficient solution has recently been proposed by Rasmussen et al. [1] when crystallizing an activated form of the human $\beta_{2}$ AR bound to a full agonist in its extracellular orthosteric binding site and to a nanobody in the intracellular side, where it mimics a $G$ protein. It is worth noting that the binding of the $G$ protein at the $\beta_{2} \mathrm{AR}$ intracellular side is able to heavily modulate the binding free energy of the isoproterenol agonist, shifting by approximately 100-fold the dissociation constant of this complex. The high RMSD value computed between $\beta_{2} \mathrm{AR}$ bound to an irreversible full agonist and uncoupled from $G$ proteins [2] and $\beta_{2} A R$ bound to a reversible full agonist and coupled to a selected nanobody (Rasmussen et al.) confirms the hypothesis that the agonist alone is not sufficient to stabilize the $\beta_{2} \mathrm{AR}$ active form. However, the above data do not completely settle this issue since it is well known that $\beta_{2} \mathrm{AR}$ is constitutively active. This peculiar behaviour has been explained by suggesting that the protein region around the binding pocket is able to efficiently sample active and inactive conformations even in the absence of a ligand [1].

Complexes of thermostabilized $\mathrm{A}_{2 \mathrm{~A}} \mathrm{AR}$ with the natural agonist adenosine (RCSB ID: 2YDO) and the synthetic agonist NECA (RCSB ID: 2YDV) were recently solved [3]. These new structures represent intermediate conformations between inactive and active states of the receptor, and are very similar to the agonist complex reported by $\mathrm{Xu}$ and colleagues [4], suggesting a common behaviour in the activation of all GPCRs.

A crystallographic structure of $\mathrm{A}_{2 \mathrm{~A}} \mathrm{AR}$ bound to an antagonist, ZM241385, was already published in 2008 [5]. The binding orientation of ZM241385 is not correctly predicted in all the previous models of $\mathrm{A}_{2 \mathrm{~A}} \mathrm{AR}$ obtained through molecular modelling based on $\beta_{2} \mathrm{AR}$ and $b \mathrm{Rh}$. Only in the X-ray structure is the antagonist oriented along the TM axis and faces the extracellular loops (ELs). However, most of the amino acids involved in ZM241385 coordination in the X-ray structure were correctly predicted.

The recently solved $\mathrm{A}_{2 \mathrm{~A}} \mathrm{AR}$ bound to the agonist UK432097 demonstrates that all the modulators based on the bicyclic adenine core scaffold bind to the same site. The overlay of heterocyclic rings of agonist and antagonist in $\mathrm{A}_{2 \mathrm{~A}} \mathrm{AR}$ and the identity of most of the amino acid interacting with the bound adenosine moiety were predicted in a modelling study [6]. Docking of agonists to inactive states of a receptor can be a valid approach, especially if constraints implied by mutagenesis are taken into account. The different intrinsic activity of agonists and antagonists is connected to their ability to specifically interact with selected residues. For instance, the ribose ring is a key feature for the agonists, and the interaction between the carbonyl O4 of UK-432097 and His ${ }^{6.52}$, not predicted through molecular modelling techniques, reveals itself as essential since $\mathrm{His}^{6.52}$ mutation disrupts agonist binding. All the collected structural data on class A GPCRs show that $\beta$-adrenergic receptors ( $\beta \mathrm{ARs}$ ) and $b \mathrm{Rh}$ binding sites share the same space location; on the contrary, $A_{2 A} A R$ ligands like ZM24138 can bind in a more superficial receptor location, suggesting that class A GPCRs can be characterized by different binding fashions.

The class A GPCR structures solved up to now point out a markedly different organization of ELs. A recent review is focused on the importance of these loops in ligand recognition and receptor activation [7]. Among these loops, EL2, the largest one, with most divergent sequence, has been proposed to act as a gatekeeper, crucial in the recognition and binding of ligands, and also involved in the allosteric modulation processes. A recent paper [8] describes significant conformational differences in the EL2 of holo- $\beta_{2}$ ARs that are connected with the intrinsic activity of the bound ligands. An 'ionic lock' between EL2 (D192) and EL3 (K305) has been identified in $\beta_{2} A R$ through NMR experiments. Its state is operated by the intrinsic activity of a bound ligand-agonists are able to weaken this interaction, stabilizing a closed conformation of the loop. This peculiar behaviour of EL2 deserves a careful investigation, and the alternative arrangement of the loop could be exploited for the pharmacological evaluation (both in silico and in vitro) of new putative class A GPCR activators. Furthermore, it is necessary to remember that a conserved motif $\mathrm{D}[\mathrm{E}] \mathrm{RY}$ in helix III and a $\mathrm{G}$ in helix VI was identified as forming an 'ionic lock' in $b \mathrm{Rh}$ and D3R bound to an antagonist $[9,10]$. This same motif, common in all class A GPCRs, has been thoroughly investigated in $\beta$ ARs and in $A_{2 A} A R$, where it seems not to be involved in the formation of any 'ionic lock', but to restrain the conformation of intracellular loop 2, which facilitates the activation of the coupled $G$ protein. In the agonist-bound state of the $\mathrm{A}_{2 \mathrm{~A}} \mathrm{AR}$, the side chains of the ionic lock residues spread far apart. This can be seen clearly in the movie file that accompanies the $\mathrm{Xu}$ et al. paper, in which the agonist- and antagonist-bound states of the receptor interconvert [4].

No need to further point out the paramount impact of solving new crystallographic structures for increasing 
the knowledgebase about molecular mechanisms and operational strategies of GPCRs. The collected data will also contribute to improve molecular modelling tools, which are at the basis of the most up-to-date rational drug design pipelines. Agonist-bound active-state GPCR structures are difficult to obtain because of the multiple conformational states the receptors explore under the activation conditions. Either the use of a high-affinity agonist [4] or binding of a suitable nanobody [1], performed as successful strategies, is certainly able to boost the state of the art in a field critical for many human health-related studies.

\section{References}

1. Rasmussen et al (2011) Nature 469:175-181

2. Rosenbaum et al (2011) Nature 469:236-240

3. Lebon et al. (2011) Nature Epub, doi:10.1038/nature10136

4. $\mathrm{Xu}$ et al (2011) Science 332:322-327

5. Jaakola et al (2008) Science 322:1211-1217
6. Ivanov et al (2009) J Med Chem 52:3284-3292

7. Peters et al (2011) TiPS 32:35-41

8. Bokoch et al (2010) Nature 463:108-112

9. Chien et al (2010) Science 330:1091-1095

10. Palczewski et al (2000) Science 289:739-745

\section{About the Authors}

Chiara Parravicini is research associate at the Department of Pharmacological Sciences at the University of Milan, Italy. She holds a $\mathrm{PhD}$ in pharmacology and her research is on purinergic pharmacology, with particular focus on the P2Y-like receptor GPR17.

Cristina Sensi is a bioinformatician at the same University and applies computational tools for solving protein structures.

Ivano Eberini is senior research associate at the Department of Pharmacological Sciences at the University of Milan, Italy. He is a molecular pharmacologist with a $\mathrm{PhD}$ in biotechnology. He is author/ coauthor of more than 65 papers, 15 of which focussed on structurefunction relationships in proteins of pharmacological interest. 